\begin{tabular}{|c|c|c|}
\hline Beitr. Ent. & Keltern & ISSN 0005-805X \\
\hline $54(2004) 2$ & S. $263-265$ & 20.12 .2004 \\
\hline
\end{tabular}

\title{
Bernhard Klausnitzer 65 Jahre!
}

Wenn Prof. Dr. sc. nat. Bernhard Klausnitzer am 21.10.04 sein 65. Lebensjahr vollendet, gilt es ein schon jetzt ungewöhnlich reiches Lebenswerk zu würdigen. Bereits als Schüler betätigte er sich entomologisch, nicht zuletzt durch den im gleichen Haus wohnenden Prof. K. H.C. Jordan angeregt und gefördert. Jordan empfahl ihm, sich nicht wie er selbst besonders mit Wanzen, sondern mit Käfern zu beschäftigen. Und diesen ist er seither treu geblieben, wobei die Coccinellidae bald in den Mittelpunkt des Interesses rückten. Damals gab es in seiner Heimatstadt Bautzen eine Gruppe sehr aktiver Entomologen, die ihm früh auch zu Begegnungen mit zahlreichen auswärtigen Entomologen verhalf. Kontaktpflege, auch über die Grenzen Deutschlands hinaus, war und ist für seine Arbeit und sein Wesen kennzeichnend. Ich erinnere mich, wie er, noch Student, 1965 beim geselligen Beisammensein anlässlich der in Dresden durchgeführten letzten der traditionellen Wanderversammlungen deutscher Entomologen wie kein anderer eine ansehnliche Zahl von Fachkollegen verschiedener Länder um sich geschart hatte, mit denen ihn schon damals freundschaftliche Beziehungen verbanden.

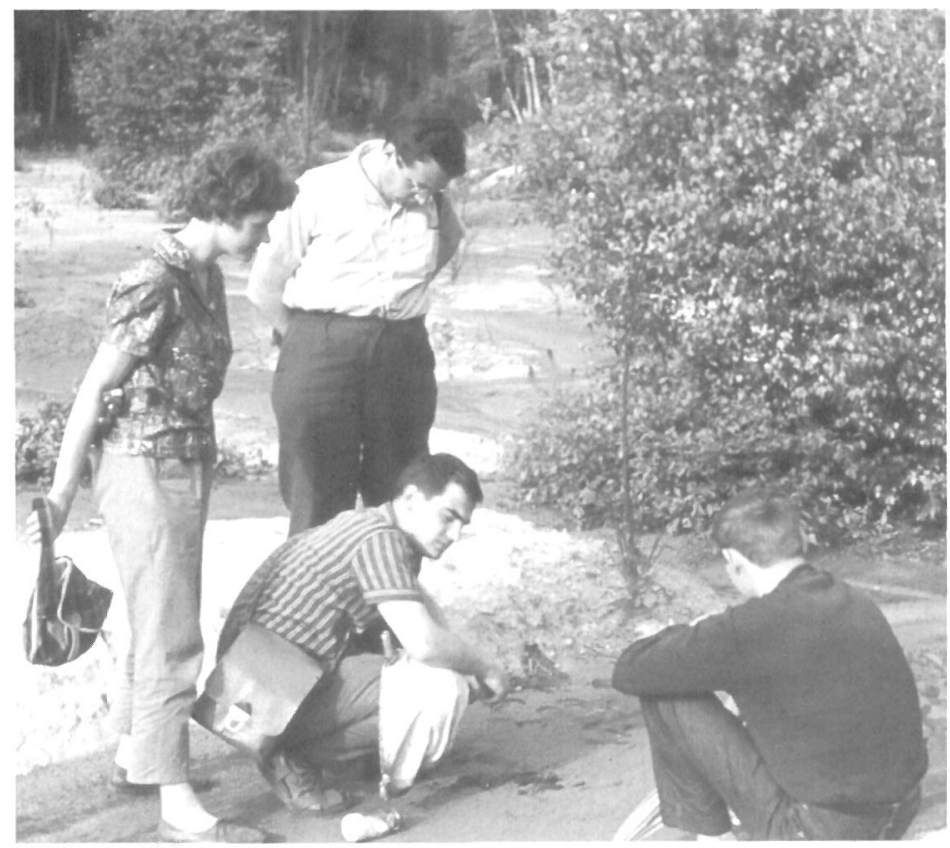

Fig. 1: Bernhard Klausnitzer als Student bei Guttau (Foto U. Sedlag)

Sein Studium absolvierte der Jubilar ab 1960 an der TH, später TU Dresden, wo er sich als „Hilfsassistent“ im Institut für Zoologie nützlich machte und dadurch auffiel, dass er ungewöhnlich aktiv in der Nutzung zusätzlicher Bildungsmöglichkeiten war, die er unter anderem durch Teilnahme an Tagungen und Gastaufenthalte in verschiedenen Institu- 
tionen suchte. Beispielsweise sei erwähnt, dass er sich zeitweilig intensiv mit Mollusken beschäftigte und an anthropologischen Grabungen teilnahm. Die Diplomarbeit (unter U. Sedlag) begründete ein besonderes Interesse für die Beziehungen zwischen Insekten und Pflanzen, das sich später in den Arbeiten seiner Schüler widerspiegelte.

1960 war Bernhard Klausnitzer bei der 9. Wanderversammlung Deutscher Entomologen erstmals mit einem Vortrag hervorgetreten, dem unzählige weitere folgen sollten. Außer fachlichen Vorträgen sind auch die mit Humor und einem umfangreichen Schatz von Zitaten gewürzten Reden erwähnenswert, die es im Lauf der Jahre bei vielen Gelegenheiten zu halten galt.

Die berufliche Laufbahn begann 1966 mit einer Assistentenstelle am Zoologischen Institut der Fakultät für Forstwirtschaft der TU Dresden in Tharandt, die sogleich den Einstieg in die schon in der Tharandter Zeit sehr vielseitige Lehrtätigkeit mit sich brachte. Forschungsobjekte waren damals naturgemäß vor allem Forstschädlinge; die an diesen gewonnenen Ergebnisse fanden überwiegend in unveröffentlicht gebliebenen Berichten ihren Niederschlag. Die Dissertation (unter H. Geiler) beschäftigte sich mit der Larvalsystematik der Coccinelliden. Sie war Ausgangspunkt für die Larvalsystematik anderer Käferfamilien, die ihre Krönung in fünf unter Mitwirkung anderer Autoren entstandenen Bänden des Freude-Harde-Lohse fand.

1977 wurde Bernhard Klausnitzer als Dozent an die Karl-Marx-Universität Leipzig berufen, an der er die akademische Laufbahn 1991 als Ordentlicher Professor (seit 1983) abschloss, d.h. abschließen musste. Schwerpunkt der dortigen Forschungstätigkeit war die noch kaum bearbeitete Urbanökologie, aus der neben eigenen zahlreiche Arbeiten von Schülern hervorgegangen sind. Sechs mit internationaler Beteiligung von 1981 bis 1989 durchgeführte Symposien zu dieser Thematik belegten die Aktualität seines Arbeitsgebietes.

Mit den Studenten verband Klausnitzer ein gutes Verhältnis. Schon in Tharandt hatte er einen Studentenzirkel Entomologie gegründet, in Leipzig betreute er jenseits des obligaten Studiums eine Interessengemeinschaft, die sich mit Fragen der Urbanökologie beschäftigte. Sein vorzeitiges Ausscheiden aus der erfolgreichen Lehrtätigkeit, in der er manchen Studenten für die Entomologie begeistern konnte, war und ist zweifellos ein großer Verlust für unser Fachgebiet. Durch seine Persönlichkeit geprägt, hatte sich in Leipzig eine Schule entwickelt, aus der weitere hochqualifizierte und -motivierte Entomologen zu erwarten waren.

Seit nunmehr fast dreizehn Jahren ist Bernhard Klausnitzer freischaffend tätig, und es ist bewundernswürdig, wie er mit ungewöhnlichem Fleiß die neue Herausforderung ohne merkliche Abstriche an seiner wissenschaftlichen Produktivität meistert. Eine Auflistung der schon über 600 Veröffentlichungen könnte den Eindruck erwecken, dass hier ein vor dem Abschluss stehendes Lebenswerk gewürdigt werden soll - es wäre zweifellos nur eine schnell an Aktualität verlierende Zwischenbilanz!

Eine solche mit 683 Titeln wurde in der aus der Feder von H. H. Dathe stammenden Würdigung in den Entomologischen Nachrichten und Berichten 48 (2004) S. 153 bis 157 veröffentlicht. (Ausführliches vom gleichen Autor ebenda 43 (1999) S. 154 bis 168.)

Allein die Zahl der Buchveröffentlichungen ist imponierend. An eine breitere Öffentlichkeit wendeten sich die Hefte der Neuen Brehm Bücherei (Marienkäfer, Hirschkäfer, Wasserkäfer, Bockkäfer, Verstädterung von Tieren), die Bücher "Wunderwelt der Käfer“ 
und „Insekten - Biologie und Kulturgeschichte", beide auch in englischer Sprache. Der zuletzt genannte Titel lässt das historische Interesse des Autors erkennen. Eine „Ökologie der Großstadtfauna" erlebte zwei Auflagen.

Damit ist die Liste der Bücher keineswegs vollständig, und darüber hinaus hat der Jubilar nicht wenige umfangreiche und wertvolle Beiträge zu Sammelwerken geliefert, insbesondere zu faunistischen. Die sechsbändige Entomofauna Germanica wird trotz Mitarbeit zahlreicher Autoren mit Recht wohl immer als sein Werk angesehen werden. Erstaunlich ist auch die als Herausgeber mehrerer Zeitschriften bewältigte Arbeit. Hier sei stellvertretend die jahrzehntelange Arbeit für die Entomologischen Nachrichten und Berichte und ihre Vorläufer genannt.

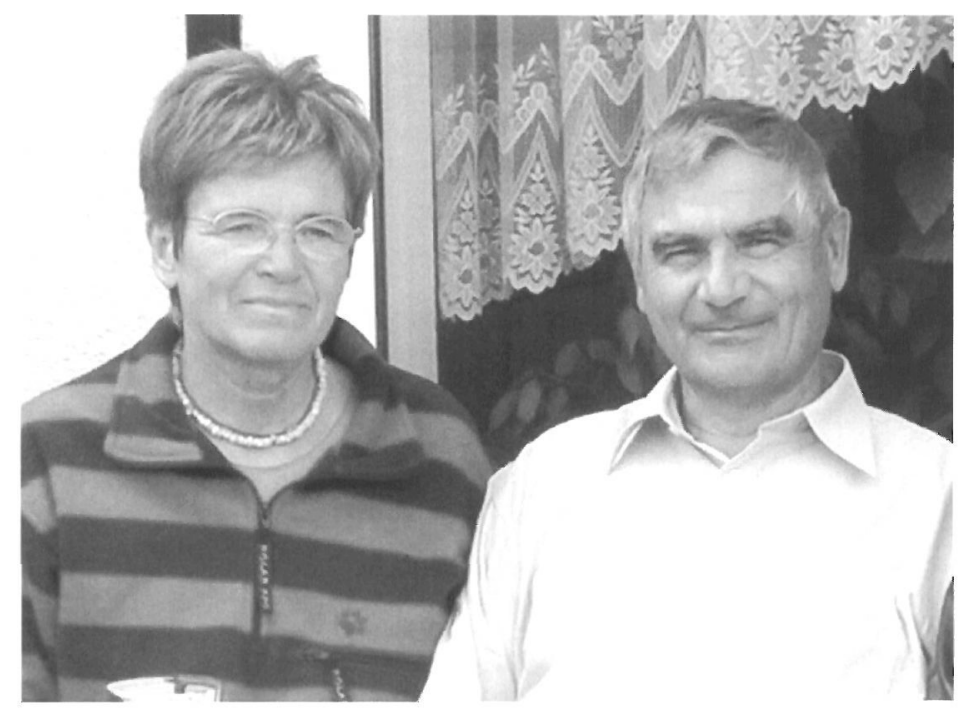

Fig. 2: Das Ehepaar Klausnitzer 2004 (Foto H. H. Dathe)

Lang ist die Liste der Organisationen, in denen Bernhard Klausnitzer, großenteils in leitender Funktion, Mitglied ist oder war, darunter sind nicht wenige ausländische. Große Verdienste hat er sich unter anderem als Mitglied und zeitweiliger Präsident des Organisationskomitees der Internationalen Symposien zur Entomofaunistik Mitteleuropas (SIEEC) erworben. 2003 wurde ihm die eigentlich nur für österreichische Entomologen gedachte Friedrich-Brauer-Medaille der Österreichischen Entomologischen Gesellschaft verliehen. Dem Deutschen Entomologischen Institut ist er seit langem verbunden, seit 2003 Vorsitzender seines Förderereins. Es ist schwer zu begreifen, wie einer unserer Kollegen ein solch immenses Arbeitspensum bewältigen konnte und kann, auch wenn man weiß, in welchem Umfang er die tatkräftige Unterstützung durch seine Frau Hertha findet.

Wenn ich dem Jubilar die besten Wünsche für Gesundheit, Anhalten der bewunderungswürdigen Tatkraft und weiteres erfolgreiches Schaffen übermittele, bin ich sicher, für viele Kollegen in aller Welt zu sprechen. 